\title{
Efecto de la temperatura superficial y la salinidad en el reclutamiento del camarón rosado Farfantepenaeus duorarum (Decapoda: Penaeidae), en la Sonda de Campeche, Golfo de México
}

\author{
Mauricio Ramírez-Rodríguez, Francisco Arreguín-Sánchez \& Daniel Lluch-Belda \\ Centro Interdisciplinario de Ciencias Marinas del IPN. Apdo. Postal 592, La Paz, 23000, Baja California Sur, México. \\ Fax: (612) 1225322; mramirr@ipn.mx
}

Recibido 20-I-2002. Corregido 04-IX-2004. Aceptado 17-III-2006.

\begin{abstract}
Impact of surface temperature and salinity on the recruiting of the pink shrimp Farfantepenaeus duorarum (Decapoda: Penaeidae), in Sonda de Campeche, Gulf of Mexico. We studied the long term effects of two environmental variables, salinity and surface temperature, on the pink shrimp (Farfantepenaeus duorarum) population in the southern Gulf of Mexico, considering the relationship between recruiting and the concurrent shrimp stock depletion of the last two decades. Our data were collected from 1969 to 1991. Recruitment has been clearly declining, particularly in the 1970s, with an accentuated drop since the 1980s. Sea surface temperatures have steadily risen, particularly since 1972. The temperature difference between the mid 1970s and the late $1980 \mathrm{~s}$ is $0.5^{\circ} \mathrm{C}$. Salinity decreased throughout the period. From a long term perspective, recruitment is negatively correlated with temperature and positively correlated with salinity. The effects of temperature and salinity are statistically significant, explaining $52 \%$ and $55 \%$ of the variation in recruitment, respectively. Rev. Biol. Trop. 54 (4): 1241-1245. Epub 2006 Dec. 15.
\end{abstract}

Key words: pink shrimp, Farfantepenaeus duorarum, Gulf of Mexico, recruitment, temperature.

La pesquería de camarón rosado Farafantepenaeus duorarum (Burkenroad, 1939) en la Sonda de Campeche, al sur del Golfo de México, registró capturas de alrededor de 20000 ton a mediados de la década de los 1970. Desde entonces presenta una tendencia decreciente, que se hizo más evidente desde 1986; desde 1990 presenta niveles de producción muy bajos, con registros inferiores a 2000 ton desde 1997. Las posibles causas del colapso se han relacionado con efectos de la sobrepesca, los cambios en el hábitat (por obras de desarrollo costero y contaminación) y la presencia de fallas en el reclutamiento (Navarrete del Próo et al. 1994, Gracia 1995, Arreguín-Sánchez et al. 1997a, b, Gracia y Vázquez-Bader 1999, RamírezRodríguez et al. 2000).
Ramírez-Rodríguez y Arreguín-Sánchez (2001) informaron que el camarón rosado presenta dos patrones estacionales de reclutamiento y dos relaciones parentela-progenie por año, que son afectadas por las condiciones ambientales en que se desarrolla cada cohorte. El efecto de la temperatura, la salinidad y otros factores ambientales en la historia de vida del camarón rosado ha sido estudiado por varios autores (Costello y Allen 1970, Bielsa et al. 1983, Hettler 1992, Criales y Lee 1995, Sheridan 1996, Gracia et al. 1997, Browder et al. 1999, Ehrhardt y Legault 1999), pero poco se ha escrito acerca de las variaciones de largo plazo de los procesos biológicos.

En este trabajo se propone la hipótesis de que los cambios a largo plazo de las condiciones ambientales podrían explicar en parte la 
disminución de la población y pesquería de $F$. duorarum en la Sonda de Campeche.

\section{MATERIALES Y MÉTODOS}

Se analiza la relación entre la magnitud del reclutamiento del camarón rosado a la pesquería de alta mar en la Sonda de Campeche y las condiciones ambientales, caracterizadas por la temperatura y la salinidad superficial del mar a lo largo del periodo de 1969 a 1991.

La magnitud del reclutamiento de individuos de tres meses de edad se estimó a través del análisis de población virtual estructurado por edades, basado en la captura por categoría comercial registrada en los puertos de Campeche y Ciudad del Carmen de 1969 a 1994 (Ramírez-Rodríguez et al. 2000, RamírezRodríguez y Arreguín-Sánchez 2001). Los datos de temperatura y salinidad fueron recabados por el Servicio Mareográfico Nacional de 1969 a 1991 en las estaciones de Ciudad del Carmen, Campeche $\left(18^{\circ} 38^{\prime} \mathrm{N}, 91^{\circ} 50^{\prime} \mathrm{W}\right)$ y Progreso, Yucatán $\left(21^{\circ} 18^{\prime} \mathrm{N}, 89^{\circ} 40^{\prime} \mathrm{W}\right)$.

Para obtener un valor representativo de toda la región, las series se promediaron mensualmente entre localidades. Posteriormente, se obtuvo el promedio anual como la media simple entre diciembre y enero. Las series estandarizadas $(\mathrm{Xsd}=(\mathrm{Xi}-\mathrm{M}) / \mathrm{ds}$, donde $\mathrm{Xi}=$ valor de la variable en el periodo $\mathrm{i}, \mathrm{M}=$ promedio, $\mathrm{ds}=$ desviación estándar de la variable en el periodo i), se sometieron a correlación lineal simple.

\section{RESULTADOS}

Los resultados del análisis de población virtual muestran que las tendencias del reclutamiento y la captura son similares. De 1969 a 1977 la tendencia de la captura fue creciente, después disminuyó lentamente hasta mediados de los ochenta y desde 1986 presentó un acelerado descenso (Fig. 1).

De 1969 a 1991 la temperatura superficial en la Sonda de Campeche tuvo una tendencia creciente, que fue más evidente desde mediados de los años 1970. La diferencia entre la temperatura a finales de los 1970 y la de finales de los 1980 fue de $0.5^{\circ} \mathrm{C}$. La salinidad presentó una tendencia creciente hasta 1982 , pero después disminuyó (Fig. 2).

El reclutamiento anual está negativamente relacionado con la temperatura superficial $(\mathrm{r}=$ $-0.72 ; \mathrm{p}<0.005 ; \mathrm{r}^{2}=0.52$ ) y positivamente con la salinidad $\left(\mathrm{r}=0.74 ; \mathrm{p}<0.05 ; \mathrm{r}^{2}=0.55\right)$. De esta forma, a medida que el ambiente se torna más caliente y menos salado se tiene un menor reclutamiento de camarón. Las ecuaciones que relacionan esas variables en los intervalos de temperatura y salinidad observados son:

$$
\begin{gathered}
\mathrm{R}=9751.7-333.45 \mathrm{SST}^{\circ} \mathrm{C} \\
\mathrm{R}=-1389.7+60.05 \mathrm{SAL}
\end{gathered}
$$

donde $\mathrm{R}=$ reclutamiento anual, $\mathrm{SST}=$ temperatura superficial promedio anual, $\mathrm{SAL}=$ salinidad promedio anual.

\section{DISCUSIÓN}

Los resultados sugieren una relación entre la disminución del reclutamiento del camarón

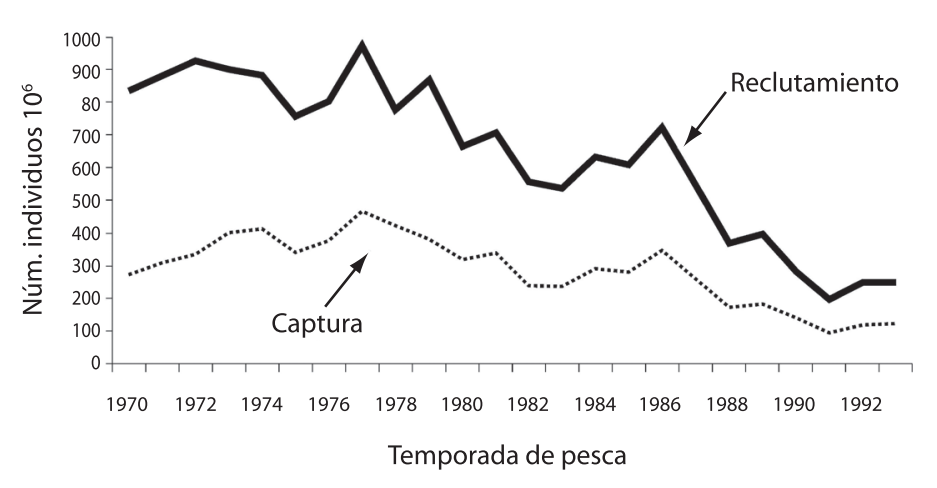

Fig. 1. Tendencias del reclutamiento y captura en número de individuos de camarón rosado F. duorarum en la Sonda de Campeche, México.

Fig. 1. Tendencies of recruitment and capture for F. duorarum in the Campeche Sound, Mexico. 

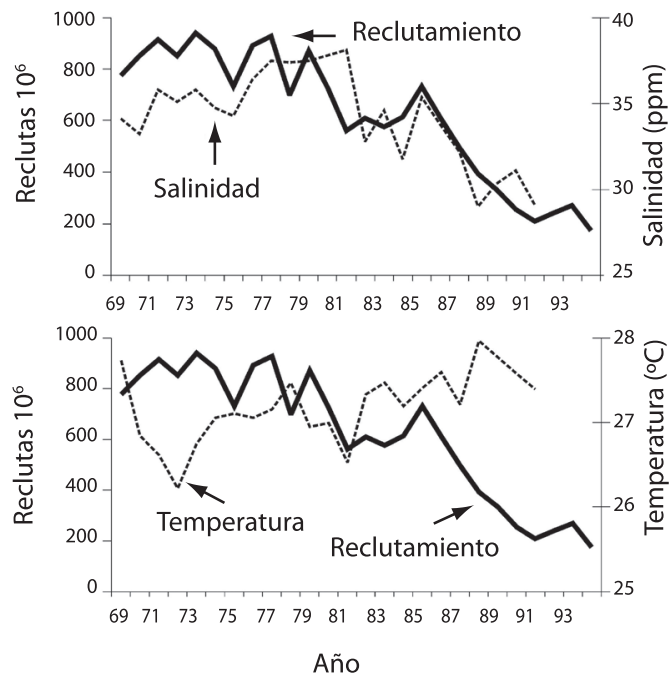

Fig. 2. Relación del reclutamiento de $F$. duorarum (línea gruesa) con la salinidad y la temperatura superficial promedio (líneas suaves) en la Sonda de Campeche.

Fig. 2. Recruitment of $F$. fuorarum (bold line), salinity and sea surface temperature (thin lines) in the Campeche Sound.

rosado en la Sonda de Campeche y las tendencias a largo plazo de aumento de la temperatura superficial y disminución de la salinidad. La linealidad de las relaciones no implica relaciones causa-efecto, sino simultaneidad de cambio. Las variaciones del reclutamiento, temperatura y salinidad son indicadores de cambio, pero por ahora se ignora cuáles pueden ser las conexiones físicas entre ellos.

La relación a corto plazo, estacional, entre cambios en la abundancia de recursos pesqueros y factores ambientales está bien documentada para diversas especies de camarones peneidos (vg. Garcia y Le Reste 1981). En escalas de mediano plazo existen evidencias de esa relación y generalmente se refieren al efecto de fenómenos climáticos como El Niño (LópezMartínez 2000). En el caso de la población de F. duorarum al sur de la Península de Florida, al norte del Golfo de México, la disminución en las capturas desde los años 1960 hasta inicios de los 90's (Sheridan 1996, Ehrhardt y Legault 1999), se relacionó con la calidad del hábitat en los estuarios y con cambios en la estructura y función del ecosistema en un periodo de décadas (Fourqurean y Robblee 1999, Hall et al. 1999, Matheson et al. 1999, Thayer et al. 1999).

En general, las evidencias sobre cambios ambientales a largo plazo en el Golfo de México indican la presencia de signos ambientales negativos principalmente en bahías, estuarios y regiones costeras (Kumpf et al. 1999) y han sido relacionados con los efectos del calentamiento global en este ecosistema (Cubit 1994, Dubravko et al. 1996, Mulholland et al. 1997. Ford (1996) y Cook et al. (1998) informaron que la temperatura superficial en invierno presenta una tendencia al aumento desde 1960, que fue más fuerte de 1986 a 1991, coincidiendo con lo encontrado en nuestro análisis.

Actualmente se desconoce cómo afectan estos cambios a los procesos físicos, químicos y biológicos descritos en el Golfo de México (vg. Salas-de-León et al. 1998, Wiseman y Sturges 1999, Lohrenz et al. 1999). Los resultados en este trabajo indican su relación con la magnitud del reclutamiento del camarón rosado y con el colapso de su pesquería en la Sonda de Campeche, por lo que los efectos de la pesca sobre la dinámica de la población y estado de la pesquería (Gracia 1995, Ramírez-Rodríguez et al. 2000) deberán analizarse en el contexto de las tendencias de las condiciones ambientales.

\section{AGRADECIMIENTOS}

Los autores agradecen al Instituto Politécnico Nacional por el apoyo parcial a través de la CGPI y de la COFAA. MRR agradece al Consejo Nacional de Ciencia y Tecnología (CONACyT).

\section{RESUMEN}

Con el objetivo de evaluar los efectos a largo plazo de variables ambientales en la población de camarón rosado Farfantepenaeus duorarum en la Sonda de Campeche, al sur del Golfo de México, se analizaron los patrones de variación del reclutamiento, la salinidad y la temperatura superficial de 1969 a 1991. El reclutamiento muestra una clara tendencia decreciente desde la década de los años 
setenta, con una disminución acentuada desde los años ochenta. La temperatura superficial muestra una tendencia al aumento, particularmente desde 1972. La diferencia entre la temperatura a mediados de los setenta y finales de los ochenta fue de $0.5^{\circ} \mathrm{C}$. A lo largo del periodo de estudio la salinidad disminuyó. En el largo plazo, el reclutamiento muestra correlación negativa con la temperatura superficial y positiva con la salinidad. Los efectos de estas variables son estadísticamente significativos, explicando respectivamente el $52 \%$ y $55 \%$ de la variación del reclutamiento $\mathrm{y}$, aunque no implican relaciones causa-efecto, sugieren simultaneidad de cambio.

Palabras clave: camarón rosado, Farfantepenaeus duorarum, Golfo de México, reclutamiento, temperatura.

\section{REFERENCIAS}

Arreguín-Sánchez, F., L.E. Schultz-Ruíz, A. Gracia, J.A. Sánchez \& T. Alarcón. 1997a. Las pesquerías de camarón de altamar: explotación, dinámica y evaluación, p. 145-172. In D. Flores-Hernández, P. Sánchez-Gil, J.C. Seijo \& F. Arreguín-Sánchez (eds.). Análisis y diagnóstico de los recursos pesqueros críticos del Golfo de México. Univ. Autón. Campeche, EPOMEX Ser. Cient. 7, Campeche, México.

Arreguín-Sánchez, F., L.E. Schultz-Ruíz, A. Gracia, J.A. Sánchez \& T. Alarcón. 1997b. Estado actual y perspectivas de las pesquerías de camarón, p. 185-203. In D. Flores-Hernández, P. Sánchez-Gil, J.C. Seijo \& F. Arreguín-Sánchez (eds.). Análisis y diagnóstico de los recursos pesqueros críticos del Golfo de México. Univ. Autón. Campeche, EPOMEX Ser. Cient. 7, Campeche, México.

Bielsa, L.M., W.H. Murdich \& R.F. Labisky. 1983. Species profiles: Life histories and environmental requirements of coastal fishes and invertebrates (South Florida) pink shrimp. Biol. Serv. Prog. Fish Wildl. Serv. (U.S.), FWS/OBS-82/11.17. 32 p.

Browder, J.A., V.R. Restrepo, J.K. Rice, M.B. Robblee \& Z. Zein-Eldin. 1999. Environmental influences on potential recruitment of pink shrimp, Farfantepenaeus duorarum, from Florida Bay nursery grounds. Estuaries 22: 484-499.

Cook, T., M. Folli, J. Klinck, S. Ford \& J. Miller. 1998. The relationship between increasing sea-surface temperature and the northward spread of Perkinsus marinus (Dermo) disease epizootics in oysters. Estuar. Coast. Shelf Sci. 46: 587-597.

Costello, T.J. \& D.M. Allen. 1970. Synopsis of biological data on the pink shrimp Penaeus duorarum duorarum Burkenroad, 1939, p. 1499-1537. In M. Mistakidis (ed.). Actas de la conferencia científica mundial sobre biología y cultivo de camarones y gambas. Cd. de México, 12-21 junio 1967. Vol. 4. Sinopsis de especies. FAO Fish. Rep. 57.

Criales, M.M. \& T.N. Lee. 1995. Larval distribution and transport of penaeoid shrimps during the presence of the Tortugas Gyre in May-June 1991. Fish. Bull. 93: 471-482.

Cubit, J.D. 1994. Global climate change and the importance of tidal flat ecosystems in the Caribbean and Gulf of Mexico. Bull. Mar. Sci. 54: 1073-1074.

Dubravko, J., N.N. Rablais \& R.E. Turner. 1996. Effects of climate change on hipoxia in coastal waters: a doubled CO2 scenario for the northern Gulf of Mexico. Limnol. Oceanogr. 41: 992-1003.

Ehrhardt, N.M. \& C.M. Legault. 1999. Pink shrimp, Farfantepenaeus duorarum, recruitment variability as an indicator of Florida Bay dynamics. Estuaries 22: 471-483.

Ford, S.E. 1996. Range extension by the oyster parasite Perkinsus marinus into the northeastern United States: Response to climate change? J. Shellfish Res. 15: 45-56.

Fourqurean, J.W. \& M.B. Robblee. 1999. Florida Bay: A history on recent ecological changes. Estuaries 22: 345-357.

Garcia, S. \& L. Le-Reste. 1981. Life cycles, dynamics, exploitation and management of coastal penaeids shrimp stocks. FAO Fish. Tech. Pap. 203, 215 p.

Gracia, A. 1995. Impacto de la pesca artesanal sobre la producción del camarón rosado Penaeus farfantepenaeus duorarum Burkenroad, 1939. Ciencias Marinas, México, 21: 343-359.

Gracia, A. \& A.R. Vázquez-Bader. 1999. Shrimp fisheries in the South Gulf of Mexico: Present state and future management alternatives, p. 205-224. In H. Kumpf, K. Steindenger \& K. Sherman (eds.). The Gulf of Mexico Large Marine Ecosystem: Assessment, Sustainability, and Management. Blackwell, Massachussetts, EEUU.

Gracia, A., A.R. Vázquez-Bader, F. Arreguín-Sánchez, L.E. Schultz-Ruíz \& J.A. Sánchez. 1997. Ecología de camarones peneidos, p. 127-144. In D. Flores-Hernández, P. Sánchez-Gil, J.C. Seijo \& F. Arreguín-Sánchez (eds.). Análisis y diagnóstico de los recursos pesqueros críticos del Golfo de México. Univ. Autón. Campeche, EPOMEX Ser. Cient 7.Campeche, México.

Hall, M.O., M.D. Durako, J.W. Fourqurean \& J.C. Zieman. 1999. Decadal changes in seagrass distribution and abundance in Florida Bay. Estuaries 22: 445-459. 
Hettler, W.F. 1992. Correlation of winter temperature and landings of pink shrimp Penaeus duorarum in North Carolina. Fish. Bull. 90: 405-406.

Kumpf, H., K. Steindenger \& K. Sherman. 1999. The Gulf of Mexico Large Marine Ecosystem: Assessment, Sustainability, and Management. Blackwell, Massachussetts, EEUU. 704 p.

Lohrenz, S.E., D.A. Wiesenburg, R.A. Arnone \& X. Chen. 1999. What controls primary production in the Gulf of Mexico?, p. 151-170. In H. Kumpf, K. Steindenger \& K. Sherman. The Gulf of Mexico Large Marine Ecosystem: Assessment, Sustainability, and Management. Blackwell, Massachussetts, EEUU.

López-Martínez, J. 2000. Dinámica de la pesquería de camarón café (Penaeus californiensis) en el litoral sonorense y su relación con algunos parámetros océano-atmosféricos. Tesis doctorado, CICIMAR, Instituto Politécnico Nacional, México. 181 p.

Matheson, R.E.J., D.K. Camp, S.M. Sogard \& K.A. Bjorgo. 1999. Changes in seagrass-associated fish and crustacean communities on Florida Bay mud banks: The effects of recent ecosystem changes? Estuaries 22: 534-551.

Mulholland, P.J., G.R. Best, C.C. Coutant, G.M. Hornberger, J.L. Meyer, P.J. Robinson, J.R. Stenberg, R.E. Turner, F. Vera-Herrera \& R.G. Wetzel. 1997. Effects of climate change on freshwater ecosystems of the southeastern United States and the Gulf Coast of Mexico, p. 949-970. In C.E. Cushing (ed.). Freshwater ecosystems and climate change. Hydrological Processes 11.

Navarrete del-Próo, A., H. Garduño-Argueta \& A. Gracia. 1994. La pesquería de camarón en alta mar. Golfo de México y el Caribe Mexicano. Instituto Nacional de la Pesca Atlas Pesquero de México. Secretaría de Pesca, CENEDIC, Universidad de Colima, México (disco compacto).

Ramírez-Rodríguez, M. \& F. Arreguín-Sánchez. 2001. Spawning stock-recruitment relationships of pink shrimp Farfantepenaeus duorarum in the southern Gulf of Mexico. Bull. Mar. Sci. 72: 123-133.

Ramírez-Rodríguez, M., E. Chávez \& F. ArreguínSánchez. 2000. Perspective of the pink shrimp (Farfantepenaeus duorarum Burkenroad) fishery of Campeche Bank, Mexico. Ciencias Marinas, México 26: $97-112$.

Salas de León, D.A, M.A. Monreal-Gomez, L. SanvicenteAnorve \& C. Flores-Coto. 1998. Long-term influence of currents on zooplanktonic organism distribution in the Bay of Campeche, Mexico. Oceanol. Acta 21: 87-93.

Sheridan, P. 1996. Forecasting the fishery for pink shrimp, Penaeus duorarum, on the Tortugas Grounds, Florida. Fish. Bull. 94: 743-755.

Thayer, G.W., A.B. Powell \& D.E. Hoss. 1999. Composition of larval, juvenile and small adult fishes relative to changes in environmental conditions in Florida Bay. Estuaries 22(2B): 518-533.

Wiseman, W.J. Jr. \& W. Sturges. 1999. Physical oceanography of the Gulf of Mexico: Processes that regulate its biology, p. 77-92. In H. Kumpf, K. Steindenger \& K. Sherman. The Gulf of Mexico Large Marine Ecosystem: Assessment, Sustainability, and Management. Blackwell, Massachussetts, EEUU. 
